\section{TATRA \\ MOUNTaiNS \\ Mathematical Publications}

DOI: $10.2478 / \mathrm{tmmp}-2021-0034$

Tatra Mt. Math. Publ. 80 (2021), 135-144

\title{
A FRACTIONAL ORDER DELAY DIFFERENTIAL MODEL FOR SURVIVAL OF RED BLOOD CELLS IN AN ANIMAL: STABILITY ANALYSIS
}

\author{
SAntoshi PAnigrahi ${ }^{1}$ — Sunita Chand ${ }^{2}$ \\ ${ }^{1}$ Department of Mathematics, Siksha 'O' Anusandhan \\ (Deemed-to-be University), \\ Bhubaneswar, Odisha, INDIA \\ ${ }^{2}$ Center for Applied Mathematics and Computing, Siksha 'O' Anusandhan \\ (Deemed-to-be University), \\ Bhubaneswar, Odisha, INDIA
}

\begin{abstract}
In this paper, we analyse stability of survival of red blood cells in animal fractional order model with time delay. Results have been illustrated by numerical simulations.
\end{abstract}

\section{Introduction}

Recently, several researchers have been working in the field of fractional differential equations due to its vast applications in various areas, such as electromagnetic field theories, control theory, fluid flow, optics, signal processing, epidemics, infectious diseases, etc. The development on fractional ordinary and partial differential equations can be found in the monograph Podlubný [1], Samko et al. [2] and the references therein.

Ważewska-Czyżewska and Lasota [3] have constructed a model for the process of generation and degeneration of red blood cells. After that many researchers have worked on the survival of red blood cells in different animals.

(C) 2021 Mathematical Institute, Slovak Academy of Sciences.

2010 Mathematics Subject Classification: 92C50, 93A30, 37N35, 34D20, 34E05, $34 \mathrm{~K} 28$.

Keyw ords: red blood cell, stability, time-delay, fractional differential equation, Caputo fractional derivative.

(c) (1) ()요 Licensed under the Creative Commons BY-NC-ND 4.0 International Public License. 
Györi and Ladas 4] have studied the asymptotic behaviour of red blood cells in animal model. Several researchers such as Song et al. [5], Fan and Wei [6] have done bifurcation analysis on survival of red blood cells in animal models. Song [7] has discussed the positive periodic solutions of a periodic survival for the red blood cell model. Lakshman and Senthilkumar [8, Dzhalladova and Růžičková [9], Sadani [10] have done the stability analysis of functional differential equations. Khalouta and Kade [1] have studied the solution of the fractional bratu-type equation by fractional residual power series method. Deng et al. [12, Čermák et al. [13, Sawoor [14] and Chartubapan et al. [15] have done the stability analysis of fractional differential equations. Several researchers such as Radha and Balamuralitharan [16] and Preethilatha et al. [17] have proposed different types of fractional order time delay biological models and have done the stability analysis of the respective models.

Li et al. 18, have studied the existence and finite time stability of a unique almost periodic positive solution for fractional order Lasota-Ważewska red blood cell models. Stamov and Stamova [19] have studied the existence, boundedness and global stability of integral manifolds for impulsive Lasota-Ważewska equations of fractional order with time varying delays and variable perturbations. Bhalekar and Daftardar-Gejji [20] have worked on a Predictor Corrector scheme for solving nonlinear delay differential equations of fractional order.

In the present work, we have analysed the stability of the fractional order model with time delay for the survival of red blood cells in animals and obtained results, which have been illustrated by some numerical simulations. Our work is motivated from the papers [3] and [20].

The paper is organised as follows:

Section 1: contains the introduction to the paper.

Section 2: we formulate the survival of the red blood cells model with time delay.

Section 3: contains the stability analysis of the model.

Section 4: we give the numerical simulation.

Section 5: contains the conclusion part of the paper.

\section{Model formulation}

Ważewska-Czyżewska and Lasota [3] constructed the following survival of red blood cells in animals model

$$
N^{\prime}(t)=-\mu N(t)+p e^{-\gamma} N(t-\tau), \quad t \geq 0,
$$

where $N(t)$ is the number of red blood cells present at time t, $\mu=$ is the rate of the red blood cells, $p$ and $\gamma$ are the production of red blood cells per unit time and $\tau$ is the time required to produce a red blood cell. 


\section{A FRACTIONAL ORDER DELAY DIFFERENTIAL MODEL ...}

In this work, we have discussed the survival of red blood cells in animals fractional order model with time delay. The model is

$$
D^{\alpha} N(t)=-\mu N(t)+p e^{-\gamma N(t-\tau)}, \quad t \geq 0
$$

with initial function

$$
N(t)=N_{0}(t), \quad-\tau \leq t \leq 0 .
$$

Equation (2) models the dynamics of the number of red blood cells at time $t$, where $\mu$ is the rate of red blood cells and $p, \gamma$ are the production of red blood cells per unit time $t$, where, $D^{\alpha} N(t)$ is the Caputo fractional order derivative and defined as follows [1]

$$
D^{\alpha} N(t)=\frac{1}{\Gamma(n-\alpha)} \int_{0}^{t} \frac{N^{(n)}(\tau)}{(t-\tau)^{\alpha+1-n}} \mathrm{~d} \tau,
$$

where $\alpha \in R, n-1<\alpha<n, n \in N$ and $\mathrm{f}$ is a continuous function.

\section{Stability analysis}

In this section, we have done stability analysis of the model (21).

Let $e^{-\gamma N(t-\tau)}=f(N(t-\tau))$. So (1) becomes

$$
D^{\alpha} N(t)=p f(N(t-\tau))-\mu N(t),
$$

where $D^{\alpha}$ represents the Caputo fractional derivative of order $\alpha$. Let $N^{*}$ be an equilibrium point of the equation (3).

Note. A point $N^{*}$ is called an equilibrium point of (3) if $N(t)=N^{*}$ is a constant solution of (3).

So

$$
p f\left(N^{*}\right)-\mu N^{*}=0 .
$$

Let us define the following transform for linearizing the model (2):

$$
\begin{aligned}
\xi=N-N^{*}, & N_{\tau}=N(t-\tau), \quad \xi_{\tau}=\xi(t-\tau), \\
D^{\alpha} \xi= & D^{\alpha} N \\
= & -\mu N(t)+p f\left(N_{\tau}\right) \\
= & p f\left(\xi_{\tau}+N^{*}\right)-\mu\left(\xi+N^{*}\right) .
\end{aligned}
$$

Note that $D^{\alpha} N^{*}=0$ because $N^{*}$ is the equilibrium point. 


\section{SANTOSHI PANIGRAHI - SUNITA CHAND}

By using Taylor's series expansion, considering only the first two terms and neglecting the higher order terms we get

$$
D^{\alpha}(\xi)=p f^{\prime}\left(N^{*}\right) \xi_{\tau}-\mu \xi
$$

which is the linearized equation [8, (2)]. The characteristic equation of (5) is obtained by applying the Laplace transform, (see [12], [17]). Thus taking the Laplace transform of (15) we get

$$
\left(\lambda^{\alpha}+\mu-p f^{\prime}\left(N^{*}\right) e^{-\lambda \tau}\right) \xi(\lambda)=p f^{\prime} N^{*} e^{-\lambda \tau} \int_{-\tau}^{0} e^{-\lambda \tau} \xi(t) \mathrm{d} t,
$$

which gives us the characteristic equation

$$
F(\lambda)=\lambda^{\alpha}+\mu-p f^{\prime}\left(N^{*}\right) e^{-\lambda \tau}=0 .
$$

If all the roots $\lambda_{i}$ of characteristic equation (6) satisfy $\operatorname{Re}\left(\lambda_{i}\right)<0, \forall i$, then an equilibrium point $N^{*}$ is asymptotically stable.

Let $\lambda=u+i v, u, v \in R\left[8\right.$. If for all the eigenvalues $\operatorname{Re}\left(\lambda_{i}\right)<0$, the corresponding solution is stable. On the other hand, even if one of the eigenvalues $\lambda$ has a positive real part, then the solution is unstable. Hence, a change in stability can occur only when the value $\lambda$ crosses the imaginary axis at

$$
\lambda=i v=v\left(\cos \frac{\pi}{2}+i \sin \frac{\pi}{2}\right), \quad v \in R .
$$

By substituting the value of $\lambda$ in (6) we get

$$
(i v)^{\alpha}+\mu-p f^{\prime}\left(N^{*}\right) e^{-i v \tau}=0 .
$$

By separating real and imaginary part of the equation (7) we obtain

$$
\begin{aligned}
& \mu+v^{\alpha} \cos \frac{\alpha \pi}{2}=p f^{\prime} N^{*} \cos v \tau, \\
& v^{\alpha} \sin \frac{\alpha \pi}{2}=-p f^{\prime}\left(N^{*} \sin v \tau\right) .
\end{aligned}
$$

By squaring both sides of equations (마)-(9) and by adding both sides of that two equation we get

$$
v^{2 \alpha}+2 \mu v^{\alpha} \cos \frac{\alpha \pi}{2}+\mu^{2}=p^{2} f^{\prime}\left(N^{*}\right)^{2} .
$$

After solving the quadratic equation (10), we get

$$
v^{\alpha}=-\mu \cos \frac{\alpha \pi}{2} \pm \sqrt{-\mu^{2} \sin \frac{\alpha \pi}{2}+p^{2} f^{\prime}\left(N^{*}\right)^{2}} .
$$

From (11), we get

$$
\tau=\frac{1}{v}\left(2 n \pi \pm \arccos \left(\frac{\mu+v^{\alpha} \cos \frac{\alpha \pi}{2}}{p f^{\prime}\left(N^{*}\right)}\right)\right), \quad n=0,1,2, \ldots
$$




\section{A FRACTIONAL ORDER DELAY DIFFERENTIAL MODEL ...}

Thus, we get the critical surfaces:

$$
\begin{gathered}
\tau_{1}(n)=\frac{\left(2 n \pi+\arccos \left(\frac{\mu+\left(-\mu \cos \frac{\alpha \pi}{2}+\sqrt{-\mu^{2} \sin ^{2} \frac{\alpha \pi}{2}+p^{2} f^{\prime}\left(N^{*}\right)^{2}}\right) \cos \frac{\alpha \pi}{2}}{p f^{\prime}\left(N^{*}\right)}\right)\right)}{\left(-\mu \cos \frac{\alpha \pi}{2}+\sqrt{-\mu^{2} \sin ^{2} \frac{\alpha \pi}{2}+p^{2} f^{\prime}\left(N^{*}\right)^{2}}\right)^{\frac{1}{\alpha}}}, \\
\tau_{2}(n)=\frac{\left(2 n \pi-\arccos \left(\frac{\mu+\left(-\mu \cos \frac{\alpha \pi}{2}+\sqrt{-\mu^{2} \sin ^{2} \frac{\alpha \pi}{2}+p^{2} f^{\prime}\left(N^{*}\right)^{2}}\right) \cos \frac{\alpha \pi}{2}}{p f^{\prime}\left(N^{*}\right)}\right)\right.}{\left(-\mu \cos \frac{\alpha \pi}{2}+\sqrt{-\mu^{2} \sin ^{2} \frac{\alpha \pi}{2}+p^{2} f^{\prime}\left(N^{*}\right)^{2}}\right)^{\frac{1}{\alpha}}} .
\end{gathered}
$$

NotE. If $\tau_{0}=\min \left\{\tau_{1}(n), \tau_{2}(n)\right\}$, then for $\tau<\tau_{0}$, the equilibrium point $N^{*}$ is asymptotically stable [17].

$N(t)$ is the number of red blood cells present at time $t$ of the model. Thus, the asymptotics of $N(t)$ as well as the equilibrium point $N^{*}$ of $N(t)$ is positive [13].

We have illustrated the same in the numerical simulation, where it can be observed from the graphs depicted on Fig 1 a) and Fig 2]a). that $N(t)$ is positive in the stable region of the system.

TheOREM 3.1. There is only one stability region for $N^{*}$ between the plane $\tau=0$ in the $(p, \mu)$ parameter space and the closest critical surface $\tau(0)$ in the $(\tau, p, \mu)$ parameter space.

Pr o of. Differentiating characteristics equation (6) with respect to $\tau$, we get

$$
\frac{\mathrm{d} \lambda}{\mathrm{d} \tau}=\frac{-\lambda\left(\lambda^{\alpha}+\mu\right)}{\alpha \lambda^{\alpha-1}+\tau\left(\lambda^{\alpha}+\mu\right)} .
$$

Now, consider the numerator of (15)

$$
\begin{aligned}
-\lambda\left(\lambda^{\alpha}+\mu\right) & =-(i v)\left((i v)^{\alpha}+\mu\right) \\
& =-v^{\alpha+1} \cos \frac{(\alpha+1) \pi}{2}-i\left(v^{\alpha+1} \sin \frac{(\alpha+1) \pi}{2}+\mu v\right) \\
& =z_{1}+i z_{2} .
\end{aligned}
$$

where,

$$
z_{1}=-v^{\alpha+1} \cos \frac{(\alpha+1) \pi}{2} \quad \text { and } \quad z_{2}=-\left(v^{\alpha+1} \sin \frac{(\alpha+1) \pi}{2}+\mu v\right) .
$$




\section{SANTOSHI PANIGRAHI - SUNITA CHAND}

Now, we consider the denominator of (15)

$$
\begin{aligned}
\alpha \lambda^{\alpha-1}+\tau\left(\lambda^{\alpha}+\mu\right)= & \alpha(i v)^{\alpha-1}+\tau\left((i v)^{\alpha}+\mu\right) \\
= & \left(\tau \mu+\alpha v^{\alpha-1} \cos \frac{(\alpha-1) \pi}{2}+\tau v^{\alpha} \cos \frac{\alpha \pi}{2}\right) \\
& +i\left(\alpha v^{\alpha-1} \sin \frac{(\alpha-1) \pi}{2}+\tau v^{\alpha} \sin \frac{\alpha \pi}{2}\right) \\
= & z_{3}+i z_{4},
\end{aligned}
$$

where

$$
z_{3}=\left(\tau \mu+\alpha v^{\alpha-1} \cos \frac{(\alpha-1) \pi}{2}+\tau v^{\alpha} \cos \frac{\alpha \pi}{2}\right)
$$

and

So,

$$
z_{4}=\left(\alpha v^{\alpha-1} \sin \frac{(\alpha-1) \pi}{2}+\tau v^{\alpha} \sin \frac{\alpha \pi}{2}\right) .
$$

$$
\begin{aligned}
\left.\left(\frac{\mathrm{d} \lambda}{\mathrm{d} \tau}\right)\right|_{\tau=\tau_{0}, v=v_{0}} & =\frac{z_{1}+i z_{2}}{z_{3}+i z_{4}} \\
& =\frac{\left(z_{1} z_{3}+z_{2} z_{4}\right)+i\left(z_{2} z_{3}-z_{1} z_{2}\right)}{z_{3}^{2}+z_{4}^{2}} \\
\frac{\mathrm{d} u}{\mathrm{~d} \tau}= & \left.\operatorname{Re}\left(\frac{\mathrm{d} \lambda}{\mathrm{d} \tau}\right)\right|_{u=0}=\frac{z_{1} z_{3}+z_{2} z_{4}}{z_{3}^{2}+z_{4}^{2}}
\end{aligned}
$$

Since

$$
0<\alpha<1, \quad v>0 \text { and } \mu>0
$$

we have

$$
\left(z_{1} z_{3}+z_{2} z_{4}\right)=\alpha v^{\alpha}\left(v^{\alpha}+\mu \cos \frac{\alpha \pi}{2}\right)>0 .
$$

Hence from equation (17), we get $\frac{\mathrm{d} u}{\mathrm{~d} \tau}>0$ on each of the critical surfaces $\tau_{1}(n)$ and $\tau_{2}(n)$. This implies that there does not exist any eigen value with negative real part across the critical surfaces (13) and (14). Also the equilibrium point is stable for $\tau=0$ when $p f^{\prime}\left(N^{*}\right)-\mu<0$. Thus, there is only one stability region enclosed by $\tau=0$ and the critical surface $\tau(0)$, closest to it.

\section{Numerical simulations}

The predictor-corrector scheme is an efficient and powerful technique for solving fractional order delay differential equations, which is a generalization of the Adams-Bashforth-Moulton method. S. Bhalekar and V. Daftardar-Gejji [20] have developed a predictor-corrector scheme in their paper for the fractional delay 


\section{A FRACTIONAL ORDER DELAY DIFFERENTIAL MODEL ...}

differential equations:

$$
\begin{aligned}
D^{\alpha} y(t) & =f(t, y(t), y(t-\tau)), & & t \in[0, T], 0<\alpha \leq 1, \\
y(t) & =g(t), & & t \in[-\tau, 0],
\end{aligned}
$$

where $D^{\alpha}$ is the Caputo fractional derivative. The corrector formula for above equation is

$$
\begin{aligned}
y_{h}\left(t_{n+1}\right)=g(0) & +\frac{h^{\alpha}}{\Gamma(\alpha+2)} f\left(t_{n+1}, y_{h}\left(t_{n+1}\right), y_{h}\left(t_{n+1}-\tau\right)\right) \\
& +\frac{h^{\alpha}}{\Gamma(\alpha+2)} \sum_{j=0}^{n} a_{j, n+1} f\left(t_{j}, y_{h} t_{j}, y_{h}\left(t_{j}-\tau\right)\right) \\
=g(0) & +\frac{h^{\alpha}}{\Gamma(\alpha+2)} f\left(t_{n+1}, y_{h}\left(t_{n+1}\right), y_{h}\left(t_{n+1-k}\right)\right) \\
& +\frac{h^{\alpha}}{\Gamma(\alpha+2)} \sum_{j=0}^{n} a_{j, n+1} f\left(t_{j}, y_{h} t_{j}, y_{h}\left(t_{j-k}\right)\right),
\end{aligned}
$$

where$$
a_{j, n+1}=\left\{\begin{array}{lll}
n^{\alpha-1}-(n-\alpha)(n+1)^{\alpha} & \text { if } & j=0, \\
(n-j+2)^{\alpha+1}+(n-j)^{\alpha+1}-2(n-j+1)^{\alpha+1} & \text { if } & 1 \leq j \leq n, \\
1 & \text { if } & j=n+1,
\end{array}\right.
$$

and the predictor formula for the fractional delay differential equation is

$$
\begin{aligned}
y_{h}\left(t_{n+1}\right) & =g(0)+\frac{1}{\Gamma(\alpha)} \sum_{j=0}^{n} b_{j, n+1} f\left(t_{j}, y_{h}\left(t_{j}\right), y_{h}\left(t_{j}-\tau\right)\right) \\
& =g(0)+\frac{1}{\Gamma(\alpha)} \sum_{j=0}^{n} b_{j, n+1} f\left(t_{j}, y_{h}\left(t_{j}\right), y_{h}\left(t_{j-k}\right)\right),
\end{aligned}
$$

where

$$
b_{j, n+1}=\frac{h^{\alpha}}{\alpha}\left((n+1-j)^{\alpha}-(n-j)^{\alpha}\right) \quad \text { and } \quad h=\frac{\tau}{k} .
$$

By using predictor-corrector scheme [20], some numerical simulations are done for the model. Let

$$
\mu=0.2, \quad b=1, \quad \gamma=1 .
$$

So we get the equilibrium point $N^{*}=1.327$. The Fig. 1 and Fig. 2 follows. 


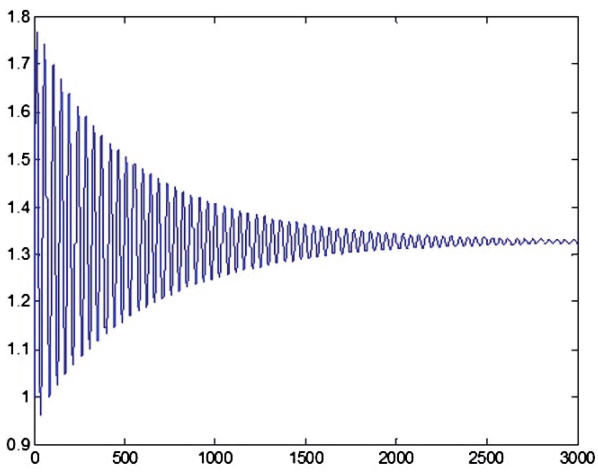

a)

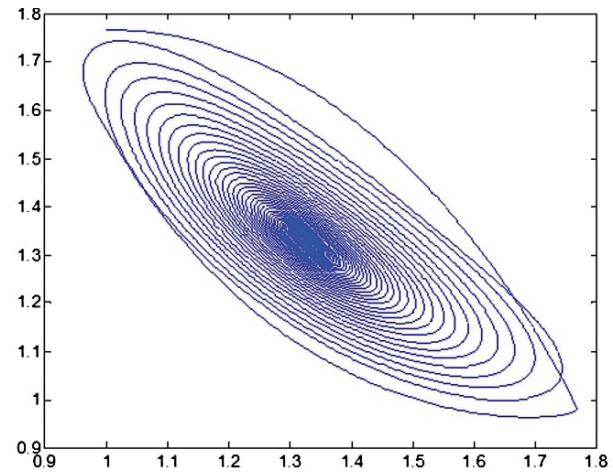

b)

FiguRE 1. Here we show the numerical simulations of fractional order model (2) when $\alpha=0.92, \tau=18$ with initial value 1 :

a) the solution $N(t)$ of the equation (2) for $\alpha=0.92$ which gives us a converging time series,

b) the stable phase portrait of the model.

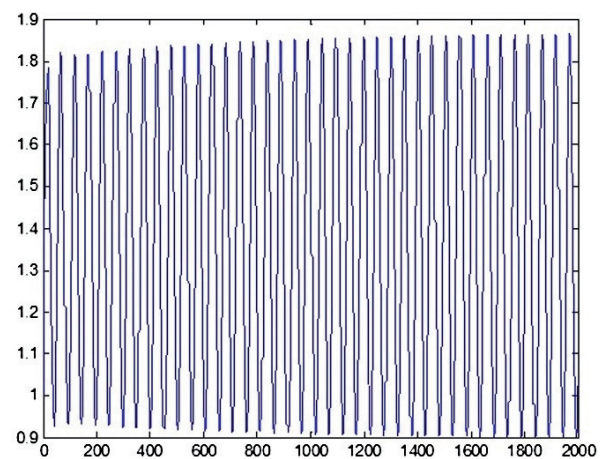

a)

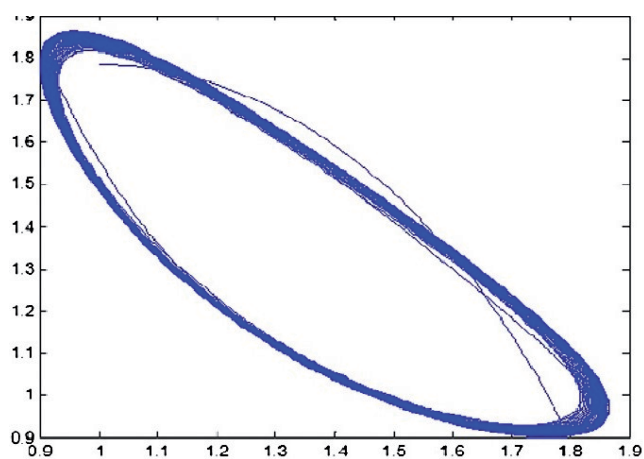

b)

FIGURE 2. There are shown the numerical simulations of fractional order model (2) for $\alpha=0.92, \tau=21$ :

a) the solution $N(t)$ of the equation (2) for $\alpha=0.92$,

b) the phase portrait of the model.

In this case, the system exhibits periodic oscillation. 


\section{A FRACTIONAL ORDER DELAY DIFFERENTIAL MODEL ...}

\section{Conclusion}

In this paper, we analysed stability of a fractional order survival of red blood cells model with time delay. The derived theoretical results have been illustrated by some numerical simulations.

\section{REFERENCES}

[1] PODLUBNÝ, I.: Fractional Differential Equations. (An introduction to fractional derivatives, fractional differential equations, to methods of their solution and some of their applications). In: Mathematics in Science and Engineering, Vol. 198. Academic Press, Inc., San Diego, CA, 1999.

[2] SAMKO, S. G.-KILBAS, A. A.-MARICHEV, O. I.: Fractional Integrals and Derivatives: Theory and Applications. Gordon and Breach Science Publishers, Yverdon, 1993.

[3] WAŻEWSKA-CZYŻEWSKA, M.-LASOTA, A.: Mathematical problems of the dynamics of a system of red blood cells. Mat. Stos. 6 (1976), no. 3, 23-40. (In Polish)

[4] GYÖRI, I.-LADAS, G.: Oscillation Theory of Delay Differential Equation: With Applications. In: Oxford Mathematical Monographs. Oxford Science Publications. The Clarendon Press, Oxford University Press, New York, 1991.

[5] SONG, Y.-WEI, J.-YUAN, Y.: Bifurcation analysis on a survival red blood cells model, J. Math. Anal. Appl. 316 (2006), 459-471.

[6] FAN, D.-WEI, J.: Bifurcation analysis of discrete survival red blood cells model, Commun Nonlinear Sci. Numer. Simul. 14 (2009), no. 8, 3358-3368.

[7] SONG, Y.: Positive periodic solutions of a periodic survival red blood cell model, Appl. Anal. 84 (2005), no. 11, 1095-1101.

[8] LAKShMAnAN, M.-SEnTHILKUMAR, D. V.: Dynamics of Nonlinear Time-Delay Systems. Springer-Verlag, Berlin, 2010.

[9] DZHAlladova, I. A.-M. RŮŽIČKOVÁ, M.: Stability of the equilibrium of nonlinear dynamical systems, Tatra Mt. Math. Publ. 71 (2018), 71-80.

[10] SADANI, I.: On the stability of the functional equation $f(2 x+y)+f\left(\frac{x+y}{2}\right)=\frac{2 f(x) f(y)}{f(x)+f(y)}+$ $\frac{2 f(x+y) f(y-x)}{3 f(y-x)-f(x+y)}$, Tatra Mt. Math. Publ. 76 (2020), 71-80.

[11] KHALOUTA, A.-KADE, A.: Solution of The fractional bratu-type equation via fractional residual power series method, Tatra Mt. Math. Publ. 76 (2020), no. 1, 127-142.

[12] DENG, W.-LI, C.-LU, J.: Stability analysis of linear fractional differential system with multiple time delays, Nonlinear Dyn. 48 (2007), no. 4, 409-416, doi: 10.1007/s11071-006-9094-0.

[13] ČERMÁK, J.-DOŠLÁ, Z.-KISELA, T.: Fractional differential equations with a constant delay: Stability and asymptotics of solutions, Appl. Math. Comput. 298 (2017), $336-350$.

[14] SAWOOR, A.AL.: Stability analysis of fractional-order linear neutral delay differential-algebraic system described by the Caputo-Fabrizio derivative, Adv. Difference Equ. 2020, paper no. 531; (2020), https ://doi.org/10.1186/s13662-020-02980-8 


\title{
SANTOSHI PANIGRAHI - SUNITA CHAND
}

[15] CHARTBUPAPAN, W.-BAGDASAR, O.-MUKDASAI, K.: A novel delay-dependent asymptotic stability conditions for differential and Riemann-Liouville fractional differential neutral systems with constant delays and nonlinear perturbation, Mathematics 8 (2020), no. 1,

https://doi.org/10.3390/math8010082

[16] RADHA, M.-BALAMURALITHARAN, S.: A study on COVID-19 transmission dynamics: Stability analysis of SEIR model with Hopf bifurcation for effect of time delay, Adv. Differ. Equ. 2020Paper no. 523 (2020), https://doi.org/10.1186/s13662-020-02958-6.

[17] PREEThilathA, V.-RIHAN, F. A.-RAKKIYAPPAN, R.-VELMURUGAN, G.: A fractional order delay differential model for Ebola infection and CD8 $+T$ cells response: Stability analysis and Hopf bifurcation, Int. J. Biomath. 10 (2017), no. 8, https://doi.org/10.1142/S179352451750111X

[18] Y. LI, Y.-WANG, Y.- LI, B.: Existence and finite-time stability of a unique almost periodic positive solution for fractional-order Lasota-Wazewska red blood cell models, Int. J. Biomath. 13, (2020), no. 2, $16 \mathrm{pp,}$ https://doi.org/10.1142/S1793524520500138

[19] STAMOV, G.-STAMOVA, I.: Impulsive delayed Lasota-Wazewska fractional models: Global stability of integral manifolds, Mathematics 7 (2019), no. 11, 15 pp, https://doi.org/10.3390/math7111025

[20] BHALEKAR, S.-DAFTARDAR-GEJJI, V.: A predictor-corrector scheme for solving nonlinear delay differential equations of fractional order, J. Fractional Calculus and Appl. 1 (2011), no. 5, 1-9, http://www.fcaj.webs.com/.

Received January 5, 2021

\author{
Santoshi Panigrahi \\ Department of Mathematics \\ Siksha 'O' Anusandhan \\ (Deemed-to-beUniversity) \\ Khandagiri Square \\ Bhubaneswar-751030 \\ Odisha \\ INDIA \\ E-mail: santoshi.panigrahi1994@gmail.com \\ Sunita Chand \\ Center for Applied Mathematics and \\ Computing \\ Siksha 'O' Anusandhan \\ (Deemed-to-be University) \\ Khandagiri Square \\ Bhubaneswar-751030 \\ Odisha \\ INDIA \\ E-mail: mami_chand@yahoo.co.in
}

